PRACTICE FORUM

\title{
How we did it: developing an antibiotic policy
}

\author{
Michael A. Borg \\ Mater Dei Hospital, Msida, Malta \\ doi:10.3396/ijic.V4i1.009.08
}

\section{Introduction}

Development of an antibiotic policy for the hospitals within the Malta Governmental Health Services followed a considerable degree of discussion, correspondence and meetings lasting several years. These centred on a wider perspective encompassing not only publication of a list of guidelines but also a broad-based approach towards sensible antibiotic prescribing. The need to improve antimicrobial prescribing, particularly in the main tertiary hospital - St. Luke's Hospital - had long been presented to the Health Department as well as the Drugs and Therapeutic Committee (DTC). A persistent increase in multi-resistant isolates had been evident throughout the previous year, culminating in Meticilin-resistant S. aureus MRSA proliferation to one of the highest incidences in Europe. Nevertheless our initiatives did not have the hoped for impact.

The breakthrough came after two unrelated developments. One was our participation in the EU network: European Study on Antimicrobial Consumption (ESAC). The publication of the first results on antimicrobial consumption patterns showed clearly that the consumption of antibiotics within hospitals in Malta was one of the highest in Europe. At the same time, the national economic situation necessitated a review of health care spending, which prompted the
Health Division to re-evaluate the expenditure for pharmaceuticals. These two events, combined with the accession of Malta into the European Union and hence a new awareness of EU recommendations of sensible antibiotic prescribing, brought about a major shift in the attitude of the Health Division.

Once departmental support had been obtained, a number of meetings were held in early 2002 between the Infection Control Committee and the DTC to achieve a consensus for a sensible antibiotic-prescribing approach. We made a holistic proposal based on a time-line of interventions including improved training at under and post graduate level, the development of guidelines for the management of infectious diseases followed by targeted protocols of stopdates for prophylaxis and restriction policies for the worst abused antimicrobials. The issue of restriction was at first a major bone of contention with the DTC representatives; however their stance softened following antimicrobial audits of carbapenems, third generation cephalosporins and glycopeptides which highlighted the level of noncompliance with evidence based recommendations.

\section{Antibiotic team}

It was agreed to form an Antibiotic Team in St. Luke's Hospital, chaired by the author, to focus predominantly on use within hospital and health centres, and a National Antibiotic Committee to serve as a forum

\section{Corresponding author}

Michael A. Borg, Mater Dei Hospital, Msida, Malta 
for discussion on all aspects of antimicrobial use and resistance within the country. The antibiotic team included another consultant microbiologist, an infectious disease physician, and the antibiotic pharmacist (we had managed to get funding for a full time pharmacist in the Infection Control Unit). Our first idea was to expand the team into a largish working group to formulate the Antibiotic Guidelines. The group would include representatives from the surgical, medical and other departments, as well as other hospital pharmacists and junior doctors.

However attempts to convene such a large group were totally unsuccessful due to the difficulty in reaching an agreed meeting time. We realised that it was going to be impossible to such a group together for more than the 20 meetings, which we predicted would be required to formulate the policy de novo. As a result, the Antibiotic Team decided to draft the policy itself but discuss each section individually with at least one or two representatives of the department which it primarily affected.

\section{Developing a policy}

The policy was printed in A5 size in order to be as userfriendly as possible. This was a compromise between some who wanted largish clear fonts and others wishing a small booklet with fine print that they could carry in their pocket. All agreed on a tabular format with recommended therapeutic options for specific medical conditions. We asked whether an outline list of antibiotics together with indications for their use would be helpful. Colleagues did not deem it to be a priority and because of restrictions in funding and a wish to keep the policy as small as possible, this was not included. We did however include costs for antimicrobials as an appendix hoping to make clinicians aware of the financial repercussions of their prescribing habits. This was well received by both the administration and the DTC.

We looked at various publications and policies and were also greatly assisted by the conclusions of the HARMONY project (http://www.harmony-microbe. net). The guidelines were set in what is now a common format with a list of infectious diseases and conditions, the likely microbial aetiology, and the recommended antibiotic. An alternative to first line therapy was given especially for individuals with a penicillin allergy. We decided at the beginning that the policy would be aimed predominantly towards the more junior members of the hospital. Therefore we did not devise specific policies for special units, which we argued would be under the responsibility of senior specialists and consultants.

We also decided that the policy should have an educational value for management of infectious disease in general. As a result, we included topics such as nomograms and aminoglycoside dosing, an extensive section dealing with the correct manner of taking microbiological specimens as well as general recommendations on proper prescribing of antibiotics. Previous audit of antimicrobial prophylaxis in surgery had indicated significant room for improvement, especially in its duration and timing. Therefore a comprehensive section was dedicated to this topic within the policy. The section on prophylaxis was also printed as a small booklet that would be available within all operation rooms in the hospital. The therapeutic choices were predominantly guided by local antimicrobial sensitivity results which were printed on the back cover of the policy. This location was chosen because it allowed us to print the table in full colour and therefore make it much easier to understand.

Once the policy was finalised, a process which took at least 40 weeks, the draft was circulated to all doctors within the health service and posted on the Infection Control Unit website. We were pleasantly surprised by the level of feedback that we obtained. Each reply was individually responded to, in some cases followed by a meeting held at the office of the individual. A considerable number of amendments were made to the original draft. Where proposals were deemed to be unacceptable, feedback was given to the individual concerned together with reasons for rejection.

\section{Looking back}

The experience of establishing a de novo antibiotic policy clearly gave an interesting insight to the subject. Although as the infection control doctor, antibiotic resistance was one of the main reasons for my efforts to 
push through this policy, it was clear, especially from administration's point of view, that at the end of the day money talks. Despite their assertions to the contrary, the message started to get across when we could actually provide figures and costings of consumption and compare them to other institutions.

We were also impressed by the initial reaction and reservation amongst professional colleagues who were quite alarmed by the concept and regarded it at first as an infringement of their freedom of choice. This was given particular importance and addressed in a number of meetings with the different departments, as well as during the annual infection control day. Partially to allay these concerns, the final policy was issued as guidelines rather than protocols to leave space for individual decision-making. Possibly stemming from this reticence, the initial reaction and lack of ownership of the situation was at the start rather discouraging. Nevertheless over the 12 months of the policy drafting, we could recognise a change in attitude, especially after a highly publicised court case involving a child who had died in hospital, and whose relatives alleged negligence in antibiotic therapy choices as the cause. Also the dissemination of our antibiotic audits helped.

We believe that acceptance will increase with time and it is hoped that by the time of the next review, ownership will have increased sufficiently. Since the work and the number of meetings that will be required on the basic document will be much less, a larger and more representative working group could be achieved to allow face to face discussion of the changes indicated.

\section{Further reading}

1. Gould IM. Antibiotic policies to control hospital-acquired infection. J Antimicrob Chemother 2008; 61: 763-5.

2. Paterson DL. The role of antimicrobial management programs in optimizing antibiotic prescribing within hospitals. Clin Infect Dis 2006; 42: S90-95.

3. Patel D, Lawson W, Guglielmo BJ. Antimicrobial stewardship programs: interventions and associated outcomes. Expert Rev Anti Infect Ther 2008; 6: 209-222.

4. MacDougall C, Polk RE. Antimicrobial stewardship programs in health care systems. Clin Microbiol Rev 2005; 18: 638656.

5. Lesprit P, Brun-Buisson C. Hospital antibiotic stewardship. Curr Opin Infect Dis 2008; 21: 344-349. 